\title{
Genome-wide analysis of single nucleotide polymorphisms in patients with atrophic age-related macular degeneration in oldest old Han Chinese
}

\author{
T.Q. Zhou, H.J. Guan and J.Y. Hu \\ Department of Ophthalmology, The Affiliated Hospital of Nantong University, \\ Nantong, Jiangsu Province, China \\ Corresponding author: H.J. Guan \\ E-mail: tqdoccn@163.com \\ Genet. Mol. Res. 14 (4): 17432-17438 (2015) \\ Received August 17, 2015 \\ Accepted October 1, 2015 \\ Published December 21, 2015 \\ DOI http://dx.doi.org/10.4238/2015.December.21.13
}

\begin{abstract}
The aim of this study was to identify disease-associated loci in oldest old Han Chinese with atrophic age-related macular degeneration (AMD). This genome-wide association study (GWAS) only included oldest old ( $\geq 95$ years old) subjects in Rugao County, China. Thirty atrophic AMD patients and 47 age-matched non-AMD controls were enrolled. The study subjects underwent a complete ophthalmic examination. Genomic DNA was extracted from peripheral blood samples. Single nucleotide polymorphisms (SNPs) were scanned by Genome-Wide Human Mapping SNP 6.0 Arrays and GeneChip Scanner 3000 7G. The results were read and analyzed by the Affymetrix Genotyping Console software. We filtered out the SNPs with a no-call rate $\geq 10 \%$, MAF P $<0.05$, and HWE $P<0.001$. The remaining 561,277 SNPs were included in the association analysis. We found that the following 2 SNPs had the highest association with atrophic AMD: rs7624556 (located on 3q24) and rs13119914 (located on 4q34.3). In conclusion, we identified two atrophic AMD-associated SNPs (rs7624556 and rs13119914) in an oldest old Han Chinese population.
\end{abstract}


This finding may lead to new strategies for screening of atrophic AMD for Han Chinese.

Key words: Age-related macular degeneration; SNP; Oldest old; Atrophic macular degeneration; Genome-wide association study

\section{INTRODUCTION}

Age-related macular degeneration (AMD) is a prevalent multifactorial disorder of the central retina and is the leading cause of vision loss in individuals over the age of 55 in developed countries (Souied et al., 2001). Approximately 8 million Americans were living with AMD as of 2008, and the number is expected to increase to 12 million by 2020 (Jager et al., 2008). In mainland China, no nationwide survey on the prevalence of AMD has yet been conducted, but sporadic epidemiological studies have reported that the prevalence of AMD in Chinese adults is slightly lower than other populations of the same age.

The advanced stages of AMD are divided into two types: atrophic (dry) AMD, which is characterized by progressive atrophy of retinal pigment epithelium (RPE) and photoreceptor cells, a loss of central vision, and slow vision loss in both eyes simultaneously; and exudative (wet) AMD, which is characterized by subretinal choroidal neovascularization leading to leaking, bleeding, and scarring with both eyes commonly involved successively and rapid loss of central vision. Atrophic AMD accounts for the vast majority of Chinese AMD cases (80-90\%).

The pathogenesis of AMD has not yet been determined. It is a complex disease caused by the combination of multiple environmental and genetic factors or interactions between them. Based on recent domestic and international research, the main risk factors for AMD are summarized as follows: decline of RPE metabolic function, aging, genetic factors, environmental impact, chronic retinal light damage, nutritional disorders, autoimmune disorders, exposure to toxic substances, and cardiovascular diseases. Research on genes related to AMD has been inconclusive because of the complexity of the disorder and involvement of multiple factors. Thus far, genes that have been reported to be associated with AMD include: $A B C A 4, C F H, E F E M P 1, C F B, C 2, R D S, V E G F$, ELOVL4, CX3CR1, HTRA1, LOC387715, VMD2, APOE, and TIMP3 (Kanda et al., 2005; Klein et al., 2005; Gold et al., 2006; Fu et al., 2007; Leveziel et al., 2007; Spencer et al., 2007; Lin et al., 2008; Chen et al., 2012; Fritsche et al., 2012; Priya et al., 2012; Stanton et al., 2012; Sun et al., 2012; Hirata et al., 2013; Ersoy et al., 2014; Nazari Khanamiri et al., 2014). Although the involvement of some of some of these genes with AMD has been demonstrated in previous studies, conflicting results have also been reported. Rugao county in Jiangsu province is well-known for its high population of senior citizens, and therefore, genome-wide SNP-based linkage scans were conducted there for atrophic AMD patients and control participants among Han individuals older than 95 years of age.

\section{MATERIAL AND METHODS}

\section{Clinical data}

Here, we performed a case-control study, and all cases and controls were recruited from Han individuals older than 95 years of age from Rugao City. Those participants who were 
in good general condition and could cooperate were enrolled and were classified into two groups: atrophic AMD group and control group. There were 30 cases of atrophic AMD, including 4 males and 26 females, with a mean age of $97.83 \pm 1.86$ years. All patients underwent eye examinations (visual acuity, slit lamp, ophthalmoscopy, OCT, etc.), and the diagnostic criteria of AREDS (age-related eye disease study) were applied. All 47 subjects in the control group were AMD-free, among which 9 were males and 38 were females, with an average age of 98.89 \pm 2.74 years. The control individuals had no family ties and no family history of AMD. Those participants who had renal insufficiency, blood diseases, diabetes, benign or malignant tumors, or other retinal choroidal diseases were ruled out. This study was conducted in accordance with the Declaration of Helsinki. This study was conducted with approval from the Ethics Committee of Affiliated Hospital of Nantong University. Written informed consent was obtained from all participants.

\section{Genome-wide SNP-based linkage scan}

Peripheral blood samples of subjects were collected, and the QIAamp DNA Blood Mini Kit (QIAGEN, Germany) was utilized to extract genomic DNA. Genome-Wide Human SNP Array 6.0 of Affymetrix Company (USA) was utilized, and the testing platform was provided by Biochip from the Shanghai National Engineering Research Center (China). The chip contained more than 1,800,000 genetic markers, 906,600 single nucleotide polymorphisms (SNPs), and more than 946,000 probes for the detection of copy number variations. Approximately 482,000 SNPs were derived from the previous-generation Mapping 500K and SNP 5.0 Arrays. The remaining 424,000 SNPs included tag SNP markers derived from the International HapMap Project, better representations of SNPs on chromosomes X and Y, mitochondrial SNPs, SNPs in recombination hotspots, and new SNPs added to the dbSNP database after completion of the Mapping 500K Array.

\section{Statistical analysis}

Scan results were read from the CEL file using Genotyping Console software. The nocall rate and minor allele frequency (MAF) of Autosomal Loci were detected. Hardy-Weinberg equilibrium (HWE) was tested, and the SNPs with a no-call rate $\geq 10 \%$, MAF $<0.05$, and HWE $\mathrm{P}<0.001$ were filtered out. The frequencies of the alleles and genotypes were calculated, and their distributions between cases and controls were compared by chi-square tests and Fisher's exact tests to assess for statistical significance. Allele frequency $P$ values obtained from chi-square tests were expressed as allele_P, and $P$ values obtained from Fisher's exact test were expressed as allele_XP. Genotype frequency $P$ values obtained from chi-square tests were expressed as genotype_P, and P values obtained from Fisher's exact tests were expressed as genotype_XP. Additionally, genotype frequencies were compared using the Cochran-Armitage trend test for cases and controls, and the $P$ values obtained were expressed as genotype_P_trend and genotype $\mathrm{XP}$ _trend, respectively. Bonferroni correction and false-discovery rate (FDR) were applied for the multiple comparison correction for allele_P, genotype_P, and genotype_P_trend. If there were no positives across the entire set of tests, then the smallest $P$ value among allele_P, genotype_P, and genotype_P_trend, called MinP, was accepted to be the main index value for scanning the single most significant SNP at each locus. 


\section{RESULTS}

\section{Sample statistics}

The control group consisted of 47 participants with a mean age of $98.89 \pm 2.74$ years. The ADM group consisted of 30 cases with a mean age of $97.83 \pm 1.86$ years. There were 26 women and 5 men in ADM group; there were 9 males and 38 females in control group. No significant differences in age and gender distribution between the two groups were found $(t=0.067, P>0.05$; $\left.\chi^{2}=0.453, P=0.501\right)$.

\section{SNP screening}

In total, there were 46,622 SNPs with a no-call rate $\geq 10 \% ; 273,088$ SNPs with MAF $<0.05$; and 166,195 SNPs with HWE P $<0.001$. After their removal, there were 561,277 remaining loci, and the chromosomal positional distribution of each locus is shown in Figure 1.

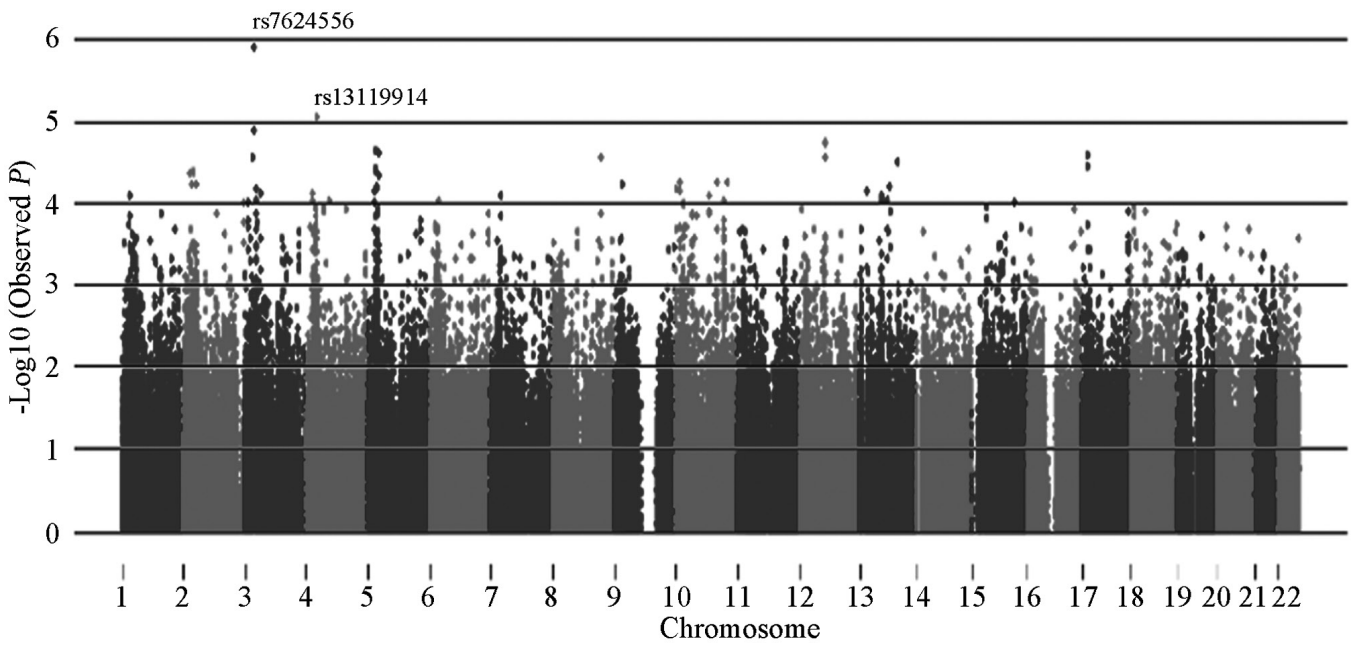

Figure 1. Distribution of 561,277 SNPs along the chromosomes after filtration. There were 776 SNP loci with MinP < $10^{-3}, 59$ SNP loci with MinP $<10^{-4}$, and 2 SNP loci with MinP $<10^{-5}$ (rs7624556 and rs13119914).

No positive results were obtained from the Bonferroni and FDR multiple comparison correction tests, and therefore, MinP was used as the index value for screening SNPs. Comparing the two groups, 776 SNPs were obtained when MinP $<10^{-3}$ was used as the scanning standard, whereas 59 SNPs were obtained when MinP $<10^{-4}$ was used as the scanning standard. Additionally, another 2 SNPs, namely rs7624556 and rs13119914, were obtained when MinP $<10^{-5}$ was chosen as the scanning standard. The top 20-ranked SNPs based on MinP values are shown in Table 1.

An additional independent sample was used in the validation study. It included 208 atrophic AMD cases and 218 non-AMD controls (NC) from the Chinese Han population. All cases and controls were recruited using uniform criteria. PCR techniques were used to screen the rs7624556 and rs13119914 polymorphisms. The differences in the frequencies of alleles and genotypes were then compared between the groups. However, no significant differences were observed for these SNPs between the AMD and NC groups for the frequencies of alleles or genotypes $(P>0.05)$. 
T.Q. Zhou et al.

17436

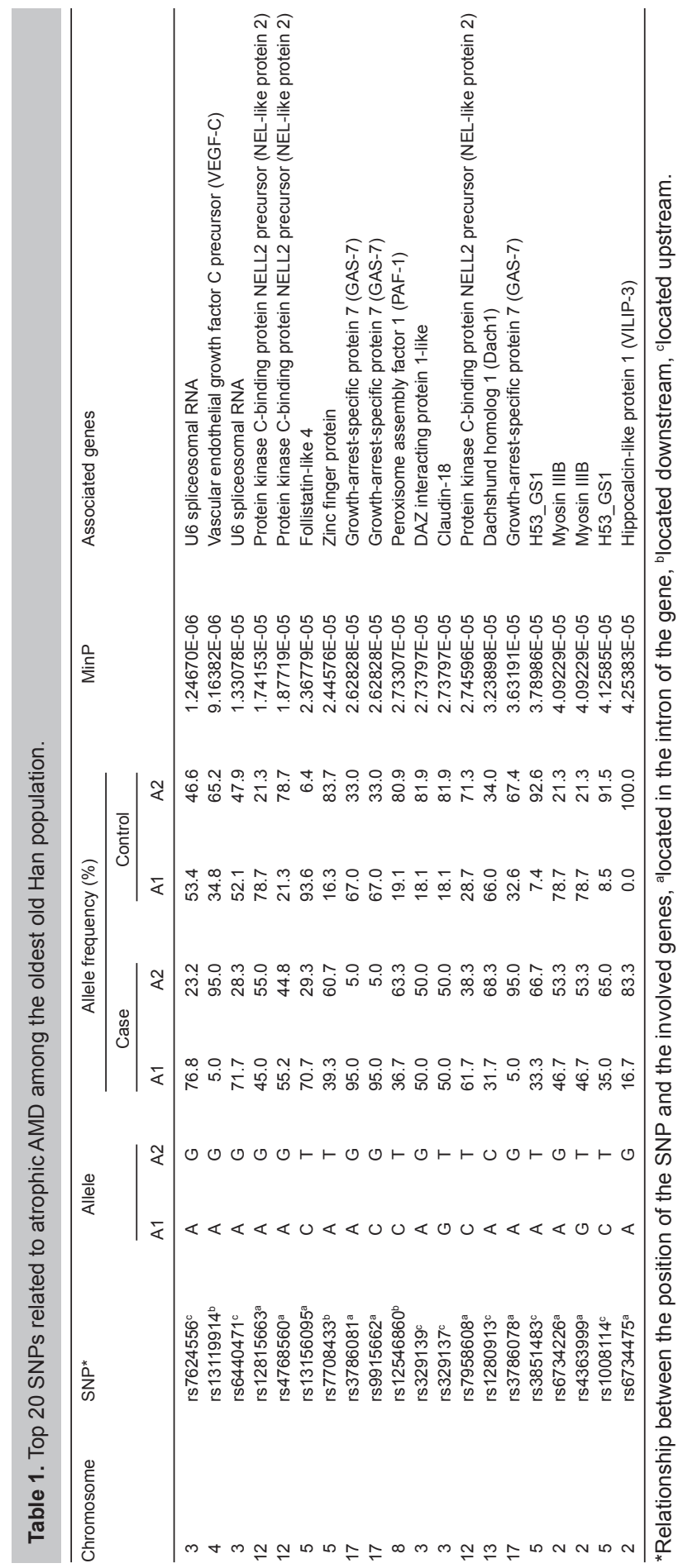

Genetics and Molecular Research 14 (4): 17432-17438 (2015) 


\section{DISCUSSION}

AMD is recognized as a complex syndrome caused by the combination of environmental and/or genetic factors. Its prevalence increases with age, and there is no difference in risk between genders. Nevertheless, clear regional and ethnic differences in disease prevalence have been observed. For example, in the United States, there is a higher prevalence of AMD among Caucasians compared to blacks, and there is a significant difference in AMD prevalence between Hispanic whites and non-Hispanic whites. Additionally, clinical sub-types of AMD differ significantly from population to population. For instance, there is a higher prevalence of atrophic AMD among Chinese, Icelandic, and Norwegian populations, whereas the prevalence of exudative AMD is comparatively higher in U.S. and Australian populations than in the European population (Björnsson et al., 2006).

As the prevalence of AMD increases with age and atrophic AMD is much more common in the Chinese Han population (accounting for $80-90 \%$ of AMD cases), atrophic AMD patients and AMD-free participants among Han individuals older than 95 years were enrolled in this study; the results were highly reliable because of the extremely low AMD incidence in the control group despite age.

There was virtually no overlap with the atrophic AMD-related gene loci obtained herein to those previously reported from both domestically and abroad, which may be because of the following aspects: special populations and small sample sizes; national and ethnic variances; and lack of validation that reported genes are actually correlated with AMD.

A decade ago, Klein et al. (2005) used gene chip technology for the first time to perform a genome-wide association study on 96 AMD patients and 50 AMD-free subjects of nonHispanic whites. Their results showed that the complement factor $\mathrm{H}(\mathrm{CFH})$ gene was closely linked to AMD.A validation study of AMD-associated genes was conducted by ophthalmic researchers in China using PCR technology (Zhao et al., 2008). Their results demonstrated that the CFH gene was associated with exudative AMD among Han population, but the opposite results have also been obtained. Similar phenomena have occurred for other purported AMDassociated genes.

Of particular note, some of the top 20 SNPs found in this analysis merit further attention. For example, a recent study on the VEGF-C gene located upstream of rs13119914 demonstrated that oxidation can stimulate increased expression of VEGF-C in the RPE (Kannan et al., 2006). The inflammatory response initiated by nuclear factor-kappa B (NF-kB)-mediated promoter activation could be a potential molecular mechanism. In future studies, the 59 SNPs obtained herein with $\mathrm{P}<10^{-4}$ will be further validated with larger sample sizes and using a custom chip.

\section{Conflicts of interest}

The authors declare no conflict of interest.

\section{ACKNOWLEDGMENTS} Corporation.

We gratefully acknowledge the technical assistance of Shanghai Biotechnology 


\section{REFERENCES}

Björnsson OM, Syrdalen P, Bird AC, Peto T, et al. (2006). The prevalence of age-related maculopathy (ARM) in an urban Norwegian population: the Oslo Macular Study. Acta Ophthalmol. Scand. 84: 636-641.

Chen W, Stambolian D, Edwards AO, Branham KE, et al. (2012). Genetic variants near TIMP3 and high-density lipoproteinassociated loci influence susceptibility to age-related macular degeneration. Proc. Natl. Acad. Sci. U. S. A. 107: 7401-7406.

Ersoy L, Ristau T, Hahn M, Karlstetter M, et al. (2014). Genetic and environmental risk factors for age-related macular degeneration in persons 90 years and older. Invest. Ophthalmol. Vis. Sci. 55: 1842-1847.

Fritsche LG, Fleckenstein M, Fiebig BS, Schmitz-Valckenberg S, et al. (2012). A subgroup of age-related macular degeneration is associated with mono-allelic sequence variants in the ABCA4 gene. Invest. Ophthalmol. Vis. Sci. 53: 2112-2118.

Fu L, Garland D, Yang Z, Shukla D, et al. (2007). The R345W mutation in EFEMP1 is pathogenic and causes AMD-like deposits in mice. Hum. Mol. Genet. 16: 2411-2422.

Gold B, Merriam JE, Zernant J, Hancox LS, et al. (2006). Variation in factor B (BF) and complement component 2 (C2) genes is associated with age-related macular degeneration. Nat. Genet. 38: 458-462.

Hirata FE, Vasconcellos JP, Medina FM, Rim PH, et al. (2013). Association of LOC387715/ARMS2 (rs10490924) gene polymorphism with age-related macular degeneration in the Brazilian population. Ophthalmic Genet. 30: 1-5.

Jager RD, Mieler WF and Miller JW (2008). Age-related macular degeneration. N. Engl. J. Med. 358: 2606-2617.

Kanda A, Chen W, Othman M, Branham KE, et al. (2007). A variant of mitochondrial protein LOC387715/ARMS2, not HTRA1, is strongly associated with age-related macular degeneration. Proc. Natl. Acad. Sci. U. S. A. 104: 16227-16232.

Kannan R, Zhang N, Sreekumar PG, Spee CK, et al. (2006). Stimulation of apical and basolateral VEGF-A and VEGF-C secretion by oxidative stress in polarized retinal pigment epithelial cells. Mol. Vis. 12: 1649-1659.

Klein RJ, Zeiss C, Chew EY, Tsai JY, et al. (2005). Complement factor H polymorphism in age-related macular degeneration. Science 308: 385-389.

Leveziel N, Souied EH, Richard F, Barbu V, et al. (2007). PLEKHA1-LOC387715-HTRA1 polymorphisms and exudative agerelated macular degeneration in the French population. Mol. Vis. 13: 2153-2159.

Lin JM, Wan L, Tsai YY, Lin HJ, et al. (2008). Vascular endothelial growth factor gene polymorphisms in age-related macular degeneration. J. Am. Ophthalmol. 145: 1045-1051.

Nazari Khanamiri H, Ghasemi Falavarjani K, Sanati MH, Aryan H, et al. (2014). Complement factor H Y402H and LOC387715 A69S polymorphisms in association with age-related macular degeneration in Iran. J. Ophthalmic. Vis. Res. 9: 181-187.

Priya RR, Chew EY and Swaroop A (2012). Genetic studies of age-related macular degeneration: lessons, challenges, and opportunities for disease management. Ophthalmology 119: 2526-2536.

Souied E, Kaplan J, Coscas G and Soubrane G (2001). Age-related macular degeneration and genetics. J. Fr. Ophtalmol. 24: 875-885.

Spencer KL, Hauser MA, Olson LM, Schmidt S, et al. (2007). Protective effect of complement factor B and complement component 2 variants in age-related macular degeneration. Hum. Mol. Genet. 16: 1986-1992.

Stanton CM, Chalmers KJ and Wright AF (2012). The chromosome 10q26 susceptibility locus in age-related macular degeneration. Adv. Exp. Med. Biol. 723: 365-370.

Sun C, Zhao M and Li X (2012). CFB/C2 gene polymorphisms and risk of age-related macular degeneration: a systematic review and meta-analysis. Curr. Eye Res. 37: 259-271.

Zhao WB, Jin XM, Bao YZ and Zhang YM (2008). The correlation between exudative AMD and CFH Y402H among the population in the central plains region of China. Ophthalmology 26: 705-707. 